Virtual Mentor. March 2004, Volume 6, Number 3.

doi: 10.1001/virtualmentor.2004.6.3.cprl1-0403

Clinical Pearl

\title{
Computer Physician Order Entry (CPOE)
}

\section{Computer physician order entry has been shown to decrease medical errors and increase the safety of patients and productivity of hospital staff.}

\author{
Donald Levick, MD, MBA
}

It is well documented in the literature that computer physician order entry (CPOE) systems can successfully reduce adverse drug events (ADE) and improve patient safety. Studies by Bates and others have shown that CPOE systems can reduce medical errors overall by up to 55 percent [1-2]. The Leapfrog Group, a coalition of more than 150 public and private organizations that provide health care benefits, has made CPOE 1 of 3 standard practices for reducing preventable mistakes in its participating hospitals [3]. Recent evidence, however, suggests that less than 5 percent of all hospitals have successfully implemented CPOE systems [4]. Cultural and work process changes present significant challenges to the successful integration of CPOE systems.

\section{The Benefits of CPOE Systems}

CPOE has been shown to decrease ADEs up to 80 percent [2]. This reduction translates into shorter lengths of stay in the hospital and lowers the average cost of a hospital admission. CPOE eliminates extra work for nurses and pharmacists - they no longer have to spend time interpreting illegible handwritten orders. Nurses, in particular, then have more time to spend with patients. Physicians are more efficient and productive because they are interrupted less frequently for interpretation of their orders.

CPOE results in quicker turnaround time for medications, ie, the time from order entry to when the medication is available to the patient. Transmission of orders to ancillary departments (radiology, laboratory) is instantaneous, leading to more efficient patient care. Finally, the system makes data easier to capture for prospective and retrospective analysis.

\section{The Challenges of Implementing CPOE}

Implementing CPOE can be difficult because it requires changing the work process and culture. Prospective users may be resistant to the new system - a problem that can be especially acute in hospital settings that do not mandate nonemployed physicians to use the CPOE system. To overcome resistance, hospitals must adequately address access to computers, user interface, and convenient training and support while they are planning to introduce CPOE, thus reducing physicians' reasons for not using the system.

Although there are significant benefits to CPOE, learning to use the system does take time and adds an additional burden to clinicians' patient care duties. The hospital must recognize this increased burden at the onset of the project and account for it in the implementation strategy. Even after the CPOE system is implemented, it still takes physicians more time to enter orders electronically than by hand. Physicians and other users must be continually reminded of the benefits of the system such as remote access, decision support (tools embedded in the computer system to aid the physician in clinical decision making, such as allergy and drug interaction alerts), fewer callbacks for interpretation of handwritten orders, and fewer ADEs. 
CPOE systems have the potential for an undesirable consequence: less verbal communication between members of the health care team, especially if remote access makes it possible for physicians to enter orders from offsite locations without discussing them with nurses or other physicians involved in the patient's care. Hospitals should adopt procedures that will allow for stat and verbal orders to mitigate this issue.

It is difficult to demonstrate the financial return on investment for a CPOE project, which costs between $\$ 5$ million to \$8 million to implement and an additional \$1 million annually to operate [5]. Although studies have shown that CPOE can reduce length of stay (which could help financial performance), it is difficult to show the direct positive financial impact of fewer ADEs. Hence, improvement in quality of care and error reduction must be emphasized as the driving forces for CPOE.

Finally, it is a challenge to integrate CPOE systems with the other clinical systems to ensure accurate and timely transmission of data. The pharmacy system is the most critical interface to address.

\section{Keys to Success with CPOE Systems}

First, there must be buy-in and commitment at all levels of the organization, starting with the board of trustees, including senior management and departmental managers. Physician leadership, from both formal leaders and informal opinion leaders, must also support the change and understand CPOE's benefits and risks and why it is being implemented.

Second, the hospital administration must elicit physician input early and often. Physicians should be involved in every phase of the process - from vendor selection to screen design and order set creation. If this is done well, the physician community will accept the new system and help create a cadre of "super-users," who facilitate use of the system by the rest of the medical staff.

Third, successful implementation requires easy access to computers. An appropriate number of devices (both fixed and wireless, if possible) must be budgeted for and maintained throughout the project.

Finally, training must be convenient for the hospital staff. Although nursing and support staff usually do well in classroom settings, physicians prefer to be trained one-on-one. The training and support staff must adopt a "wherever, whenever" attitude. Web-based or computer-based training can also be considered as possible supplemental training tools. Easily accessible support provided by knowledgeable people must be available to hospital staff newly trained in the CPOE system. The standard IS support group may not be prepared to handle clinical or detailed questions regarding the system. On-site support during the initial phases of implementation is also important to maintain momentum among the users. Ongoing 24/7 support should be provided as new physicians come on board and previously trained physicians require continued assistance.

\section{References}

1. Kohn LT, Corrigan JM, Donaldson MS, eds; Committee on Quality of Health Care in America, Institute of Medicine. To Err is Human: Building a Safer Health System. Washington DC: National Academies Press; 2000. Google Scholar

2. Bates DW, Teich JM, Lee J, et al. The impact of computerized physician order entry on medication error prevention. J Am Med Inform Assoc. 1999;6:313-21. View Article PubMed Google Scholar

3. The Leapfrog Group Web site. Survey results. Accessed February 17, 2004.

4. American Hospital Association. AHA Guide to Computerized Order Applications. Washington, DC: American Hospital Association; 2000.

Google Scholar

5. Clinical Advisory Board. Computerized physician order entry-lessons from pioneering institutions. 
Washington, DC: Clinical Advisory Board; 2001.

Don Levick, MD, MBA, is the physician liaison information services and president elect of the medical staff at Lehigh Valley Hospital.

The viewpoints expressed on this site are those of the authors and do not necessarily reflect the views and policies of the AMA.

(C) 2004 American Medical Association. All Rights Reserved. 